\title{
Spatially Opponent Excitation and Inhibition in Simple Cells of the Cat Visual Cortex
}

\author{
David Ferster \\ Department of Neurobiology and Physiology, Northwestern University, Evanston, Illinois 60208
}

The receptive fields of simple cells in the cat visual cortex are, by definition, divided into ON and OFF subfields. There is little doubt that each subfield is generated by excitatory input from geniculate neurons of the appropriate center type: ON subfields by ON-center cells, and OFF subfields by OFFcenter cells. In intracellular records, ON subfields can be detected as regions in which light elicits a barrage of EPSPS, while in OFF subfields, turning a light off does the same. In addition, visual stimuli can evoke strong IPSPs, but these IPSPs have a receptive field spatially opponent to that of the EPSPS: Inhibition is evoked by turning a light off in an ON region and turning a light on in an OFF region. This inhibition probably arises from other cortical simple cells, and may contribute to such receptive-field properties as antagonism between subfields, binocular disparity sensitivity, and orientation selectivity.

How do cells of the mammalian visual cortex acquire their receptive-field properties? The extraordinary selectivity of cortical neurons surely depends on the visual responses of individual sources of synaptic input, and on the integration of those inputs on the membrane of the postsynaptic cell. In simple cells, 2 major synaptic inputs are evoked by electrical stimulation of the lateral geniculate nucleus (LGN): a direct, monosynaptic excitation and a cortically mediated disynaptic inhibition (Toyama et al., 1974; Ferster and Lindström, 1983). Of these 2 inputs, the excitation is best understood. Its source, the relay cells of the LGN, is reflected in its monosynaptic latency. Its function, the creation of elongated $O N$ and OFF subfields, was derived from the receptive-field properties of simple cells themselves (Hubel and Wiesel, 1962), and has been supported by cross-correlation experiments (Tanaka, 1983). Tanaka found that a geniculate neuron was excitatory to a simple cell only if the receptive-field center of a geniculate neuron overlapped a simple cell subfield of the same type. There is little doubt that synaptic input from $O N$-center geniculate cells underlies $O N$ subfields and that synaptic input from OFF-center cells underlies OFF subfields.

Analogous information about inhibitory synaptic potentials in simple cells is harder to come by. On the presynaptic side, the source of the potentials, specific inhibitory interneurons, are difficult to identify and study among their countless neighbors

\footnotetext{
Received Apr. 7, 1987; revised Sept. 1, 1987; accepted Sept. 11, 1987.

This work was supported by a grant from the National Eye Institute (EY04726) and by the Alfred P. Sloan Foundation. The author is grateful for comments on the manuscript by Drs. David Van Essen, Randolph Blake, and Lawrence Pinto.

Correspondence should be addressed to Dr. Ferster at the above address.

Copyright $(1988$ Society for Neuroscience 0270-6474/88/041172-09 $\$ 02.00 /$
}

(though see Toyama et al., 1981). On the postsynaptic side, the effect of the inhibition on the behavior of simple cells is difficult to discern from extracellular records, which reflect the integration of all synaptic inputs, inhibitory and excitatory: an increase in the one can easily be taken for a decrease in the other. In some cases it is possible to identify inhibitory effects pharmacologically. GABA antagonists iontophoresed in the cortex, for example, lead to a rapid and profound loss of direction selectivity (Sillito, 1977). Alternatively, as I have done here, one can study the properties of inhibitory interneurons by recording intracellularly the IPSPs they produce in simple cells. Such records necessarily reflect the summed influence of numerous inhibitory inputs, but have the advantage that they can be compared with the properties of excitatory inputs recorded concurrently.

In a previous paper, I reported that the orientation selectivity of IPSPs recorded in simple cells matches that of EPSPs (Ferster, 1986). But while maximal EPSPs and IPSPs are evoked at identical stimulus orientations, they are not evoked at the same time or within the same region of the receptive field. The rccords presented here indicate that excitation and inhibition are spatially opponent within the simple cell receptive field. That is, in ON regions, where turning a light on evokes a synaptic excitation, turning a light off evokes a synaptic inhibition, while in OFF regions just the opposite is true.

Hubel and Wiesel (1962) first proposed the existence of spatially opponent inhibition to explain the antagonism between subfields that in part defines simple cells. They suggested that $O N$ inhibition in an OFF region would reinforce the withdrawal of excitation by geniculate OFF-center cells that results from turning a light on. The presence of this type of inhibition is supported by Heggelund's (1986) observation that the suppression of activity evoked in an OFF region at light-on is uncorrelated in strength to the discharge evoked at light-off; a single mechanism, excitation from OFF-center cells, is therefore insufficient to account for both the OFF response and the suppression at light-on. The intracellular records demonstrate the presence of spatially opponent inhibition directly. While the properties of the IPSPs suggest strongly that they arise from other simple cells in area 17, as proposed by Palmer and Davis (1981), the function of the inhibition is more a matter for speculation. IPSPs could equally well contribute to disparity sensitivity, spatial frequency selectivity, or oricntation selectivity in simple cells, or it could be critical in shaping the time course of a simple cell's response to brief stimuli.

\section{Materials and Methods}

Methods for intracellular recording have been described in detail in 2 previous papers (Ferster, 1986, 1987). Cats were anesthetized with so- 


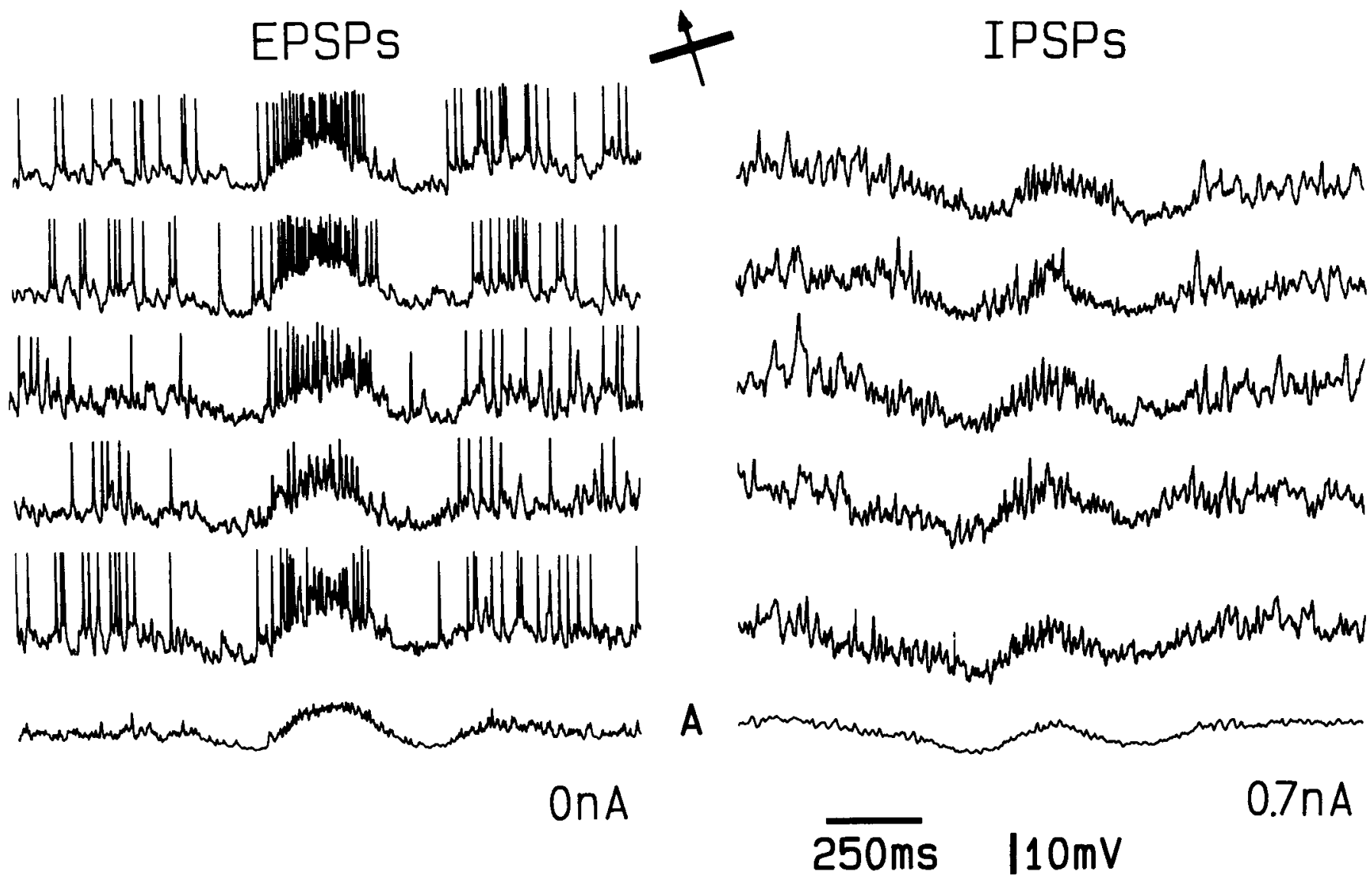

Figure 1. Intracellularly recorded responses of a simple cell evoked by a bright bar swept across its receptive field. The inset indicates the orientation and direction of motion of the stimulus. The bar measured $0.75^{\circ} \times 6^{\circ}$ and traveled $4^{\circ}$ in the $2 \mathrm{sec}$ recorded in each trace. Whether EPSPs or IPSPs were visible in the records was controlled by the amount of current injected into the cell through the recording electrode. In this and all subsequent figures, the amount of current injected into the cell through the recording electrode while recording each set of traces is indicated to the lower right. EPSPs are shown to the left $(0 \mathrm{nA})$, IPSPs to the right $(0.7 \mathrm{nA})$. The bottom trace in each column $(A)$ represents an average of 10 individual records.

dium thiopental, paralyzed with gallamine triethiodide, and artificially respirated. The eyes were focused with contact lenses of the appropriate curvature onto a tangent screen on which high-contrast bars of light were moved or flashed by a microprocessor-controlled optic bench. All stimuli were monocular. Cortical cells were penetrated with micropipettes of 50-100 M $\Omega$ resistance filled with either $4 \mathrm{M} \mathrm{K}^{+}$-acetate (Figs. $5,6)$ or $2 \mathrm{M} \mathrm{Ce}^{+}$-acetate (Figs. 1-4). $\mathrm{Ce}^{+}$is known to block voltagesensitive $\mathrm{K}^{+}$channels and increase membrane resistance (Johnston et al., 1980). Intracellular potentials were low-pass filtered and digitized (visually evoked potentials at a rate of $4 \mathrm{kH}<$; electrically evoked potentials at $20 \mathrm{kHz}$ ), and stored by computer together with information about the stimulus applied and the current injected through the recording electrode. Electrical stimulation of the LGN was delivered through a lacquer-coated tungsten electrode placed at the top of layer $\mathrm{A}$, near the representation of the area centralis.

The experiment depends critically on the separation of EPSPs and IPSPs in the intracellular records. This separation was accomplished by polarizing the membrane with DC current injected through the intracellular recording electrode. Depolarizing current shifts the membrane potential toward the equilibrium potential for EPSPs, reducing the driving force for excitatory synaptic currents, and thereby reducing the amplitude of evoked EPSPs. At the same time, the driving force on inhibitory synaptic currents, and therefore the amplitude of IPSPs, is increased. Similarly, hyperpolarizing current decreases IPSP amplitudes and increases EPSP amplitudes.

To separate EPSPs and IPSPs cleanly, the current injection must be set to the appropriate levels. Ideally, one would like to examine individual potentials and adjust the current until one type disappears, while the other is visibly increased in amplitude. It is difficult to do so, however, while examining spontaneous or visually evoked potentials; individual EPSPs and IPSPs are difficult to identify in the complex pat- terns of overlapping potentials that occur in almost random sequence. But in records evoked by stimulation of the LGN, EPSPs and IPSPs can easily be distinguished by their reproducible latencies and time courses. It is then a simple matter to adjust the current while repetitively stimulating the LGN; the effects of the current are immediately observable. In Figure 2, for example, with no current injected into the cell (Fig. $2 A$ ), only a large monosynaptic EPSP is visible. A depolarizing current of $0.7 \mathrm{nA}$ (Fig. 2C) suppresses this EPSP and reveals a disynaptic IPSP. At intermediate levels (Fig. $2 B$ ), both potentials are present, though with diminished amplitudes. The current levels arrived at while examining the electrically evoked potentials (in this case 0 and $0.7 \mathrm{nA}$ ) are then used to record the visually evoked potentials. In doing so, the implicit assumption is made that the synaptic potentials evoked by visual and electrical stimulation are identical, or at least are affected identically by the injected current. The change in the DC membrane potential necessary to suppress EPSPs significantly is likely to be $40 \mathrm{mV}$ or more, making the input resistance of the cell at least $50 \mathrm{M} \Omega$.

\section{Results}

Figures 1-4 contain intracellular records obtaincd from a simple cell in layer 4 of area 17. As explained in detail in Materials and Methods, in this cell IPSPs were recorded with $0.7 \mathrm{nA}$ injected through the electrode, while EPSPs were recorded in the absence of any injected current. These current levels were decided upon by examining the response to electrical stimulation of the LGN (Fig. 2): without current, only the monosynaptic EPSP and a small spike were visible (Fig. $2 \mathrm{~A}$ ), while $0.7 \mathrm{nA}$ of current was required to suppress the EPSP and make the di- 


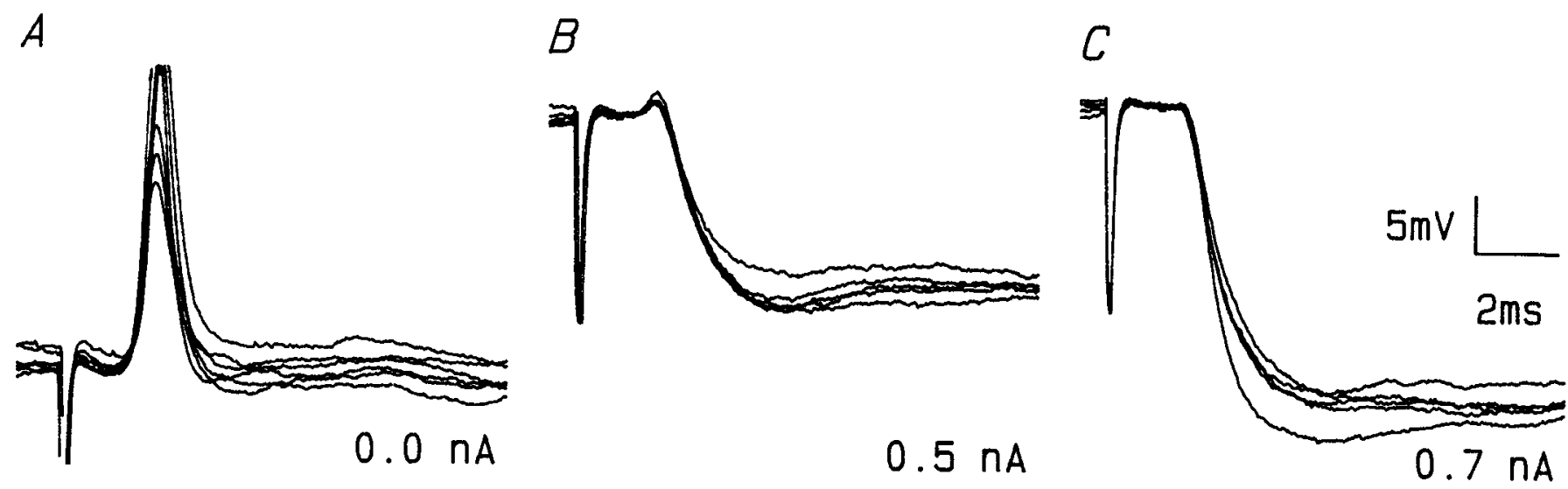

Figure 2. Response of the same simple cell as in Figure 1 to electrical stimulation of the LGN. $A$, When no current is injected through the electrode, only a monosynaptic EPSP and evoked spike are visible. These records suggest that the visually evoked records to the left of Figures 1 , 3 , and 4 , which were taken at the same level of current injection, contain predominantly EPSPs. $B$, With the cell depolarized by the injection of $0.5 \mathrm{nA}$ of DC current, the spike and the size of the EPSP is greatly reduced. A disynaptic IPSP has now appeared. $C$, An increase in the injected current to $0.7 \mathrm{nA}$ completely suppresses the EPSP and enlarges the IPSP. These records suggest that the records to the right of Figures 1 , 3 , and 4 , which were also recorded during the injection of $0.7 \mathrm{nA}$ of current, contain predominantly IPSPs. The stimulus consisted of $1 \mathrm{~mA}$ pulses of cathodal current of $200 \mu \mathrm{sec}$ duration.

synaptic IPSP visible (Fig. 2C). This IPSP had an unusually long time of decay, which is associated with the presence of $\mathrm{Ce}^{+}$ in the electrode. The current that depolarizes the membrane also injects $\mathrm{Ce}^{+}$, which presumably blocks $\mathrm{K}^{+}$channels, raises the membrane resistance, and increases the membrane time constant. Without $\mathrm{Ce}^{+}$, IPSP decay times are consistently shorter (see Fig. 6).

Once the appropriate current levels were established, the electrical stimulus was turned off, and visual stimuli were presented, first by hand to establish the position and orientation of the receptive field, and then mechanically to obtain the records of Figures 1, 3, and 4. In each of these figures, EPSPs are shown to the left, and IPSPs to the right. The level of current used to collect each set of traces is indicated below. For each of the 2 current levels, 5 individual records evoked by an identical visual stimulus are included to show the variability of the responses. Below each set of 5 are averages (A) of 10 individual traces: the 5 shown together with 5 others.

\section{Spatial organization of the EPSP receptive field in a simple cell}

Because of its response to visual stimulation, the first cell to be considered (Figs. 1-4) was identified as having a simple receptive field with 3 subregions. When a bright bar was swept across the cell's receptive field with no current applied through the electrode (Fig. 1, left), the response consisted of 4 distinct phases. In order, they were (1) a $4 \mathrm{mV}$ hyperpolarization from rest lasting $250 \mathrm{msec}$, (2) a $6 \mathrm{mV}$ depolarization from rest lasting $300 \mathrm{msec}$, (3) a second hyperpolarization, identical in size and duration to the first, and (4) a small, $2 \mathrm{mV}$, depolarization, most readily visible in the averaged trace. (This last depolarization is best secn by placing a straight cdge across the endpoints of the averaged trace.) Note that these modulations of the membrane potential evoked changes in the frequency of action potentials. While most simple cells are silent in the absence of a visual stimulus, this cell fired action potentials at rest, either because of the damage caused by the penetration or of the inactivation of $\mathrm{K}^{+}$channels by the presence of $\mathrm{Ce}^{+}$in the recording electrode. Depolarization increased the frequency of these action potentials; hyperpolarization decreased the frequency.
The 2 phases of hyperpolarization evoked by the moving stimulus are most easily interpreted as reflecting the presence of 2 OFF subfields. As the bar entered these subfields, it presumably entered the receptive-field centers of the OFF-center geniculate cells that generated the subfields. The spontaneous activity of these presynaptic geniculate neurons would be suppressed by a bright stimulus in their field centers, and the resulting withdrawal of excitation in the simple cell would cause the observed hyperpolarization. Note that the hyperpolarization must largely reflect a withdrawal of excitation, not an increase in inhibition; the records of Figure $2 A$ indicate that IPSPs did not produce a significant hyperpolarization in the absence of injected current.

The large central depolarization in Figure 1 signals the presence of a central ON region between the 2 OFF regions. Presumably the slit evoked the depolarization by entering the receptive-field centers of presynaptic ON-center cells of the LGN. (Though at the same time the slit was also leaving the centers of the OFF-center cells associated with the first OFF region.) The second depolarization, the final phase of the response in Figure 1, represents the OFF response associated with the stimulus leaving the second OFF region. That this depolarization does not reflect the presence of a second $\mathrm{ON}$ region can be deduced from the response to the bar being swept across the receptive field in the opposite direction (not shown). The first component of the response to an oppositely directed stimulus was a large hyperpolarization, associated with the slit entering the second OFF region. No depolarization preceded the hyperpolarization, as it would have if a second $\mathrm{ON}$ region were present. The full response to the oppositely directed bar closely resembled the traces of Figure 1, complete with all 4 phases, indicating that the receptive field was mirror-symmetric.

Flashing a slit in the receptive field of the cell also revealed 3 distinct subfields. To obtain the EPSP records of Figure 3, the slit was flashed in the central ON subfield, that is, the region in which the moving slit evoked the large depolarization of Figure 1. Turning the slit on in this region evoked a $4-5 \mathrm{mV}$ depolarization from rest, with a latency of $40 \mathrm{msec}$. When the slit was turned off, the cell was hyperpolarized nol only back to the resting level, but beyond it by several millivolts (latency, 45 


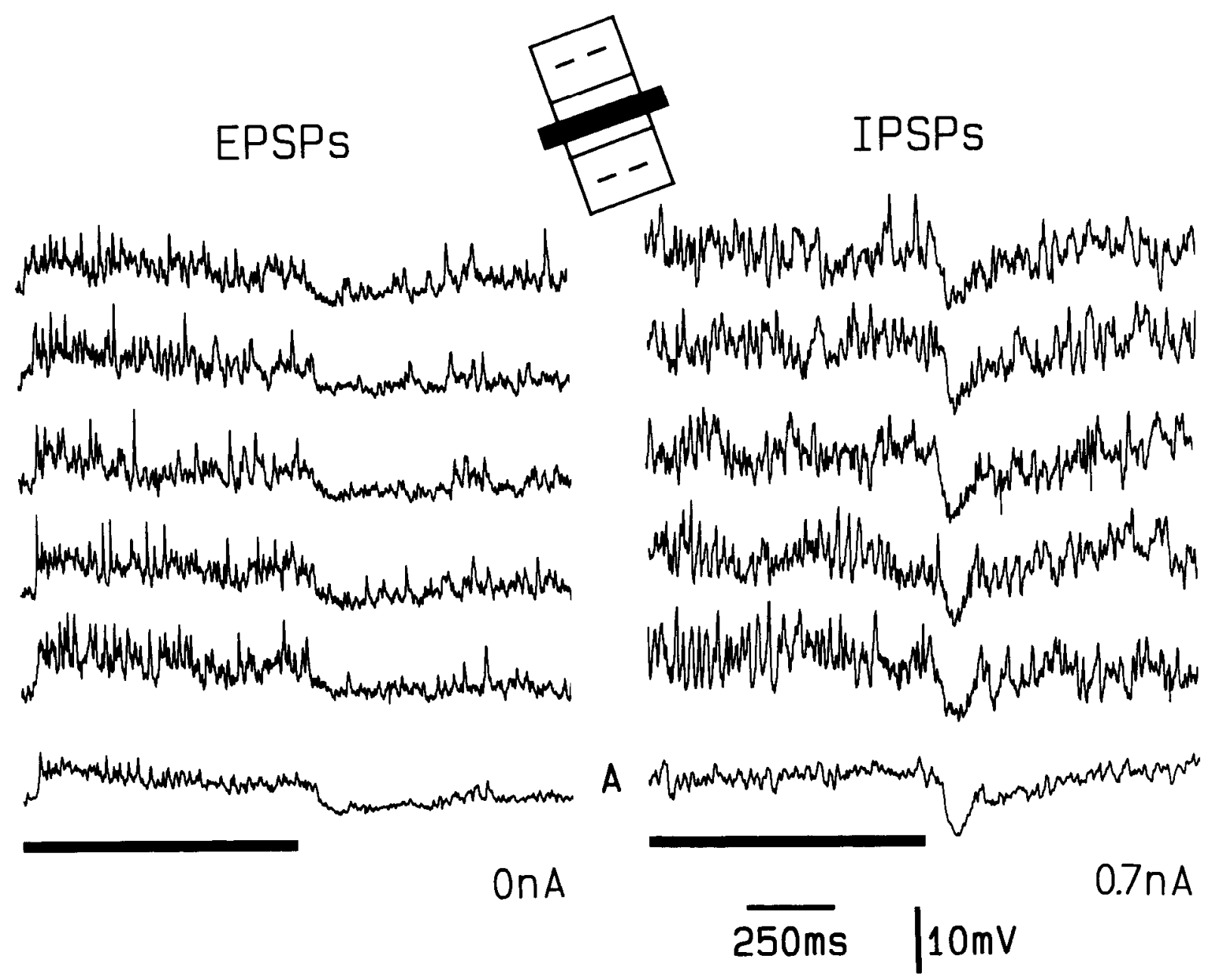

Figure 3. Responsc of the same simple cell as in Figures 1 and 2 to an optimally oriented bar flashed in the central ON region. The lines beneath the traces indicate the duration of the flash. The bottom trace of each set $(A)$ is an average of 10 individual records. As in Figure 1 , the current injected through the electrode during the collection of each set of traces is indicated to the lower right.

msec). Here again, it is assumed that the hyperpolarization evoked at light-off must represent a withdrawal of excitation (rather than an increase in inhibition), which, in turn, reflects a decrease in activity of the presynaptic cells to a level below their resting discharge. The EPSP records of Figure 3 closely resemble the modulations of spike frequency above and below resting levels evoked in geniculate $\mathrm{ON}$-center cells by flashing stimuli (Steinberg, 1983; Cleland and Lee, 1985; Cleland et al., 1971).

In a similar way, EPSPs cvoked by flashing a stimulus in either of the 2 OFF regions (Fig. 4, left) resemble the activity evoked in geniculate OFF-center cells by a stimulus flashed in their receptive-field centers: a depolarization was evoked at lightoff and a hyperpolarization at light-on. Note that the latency of the depolarization evoked from the OFF subfield at light-off, $40 \mathrm{msec}$, matched that of the depolarization evoked from the ON region at light-on. The latency of the hyperpolarization, 45 msec, also matched that of its counterpart in Figure 3. The latency of each response, therefore, depended on its own polarity, either hyperpolarizing or depolarizing, and not on that of the stimulus that evoked it, whether light-on or light-off.

Spatial organization of the IPSP receptive field in a simple cell The right-hand records of Figures 1, 3, and 4, made with the cell depolarized, show that the IPSP receptive field in this simple cell is exactly spatially opponent to the receptive fields of the EPSPs. That is, any stimulus that evoked a withdrawal of excitation also evoked an increase in inhibition, while any stimulus that evoked an increase in excitation was accompanied by a withdrawal of inhibition. In Figure 1 this organization is particularly evident for the 2 OFF regions. As the bar entered each of these OFF regions, a strong hyperpolarization was observed. In the presence of the depolarizing current, each hyperpolarization represented the activation of a true synaptic inhibition. Normally this inhibition must be evoked concurrently with the withdrawal of excitation evoked in OFF regions (visible in the EPSP records to the left), but here the 2 responses were separated in the 2 sets of records by the application of injected current.

The IPSPs evoked by flashing a stimulus in either of the OFF regions occurred at exactly the opposite phase from the EPSPs' (Fig. 4); while EPSPs were evoked when the light came on, IPSPs were evoked when the light was turned off. The 2 types of potential, therefore, reinforce each other, and the membrane potential is never allowed to return passively to rest-it is actively pulled either up or down, depending on the phase of the stimulus. Opponent organization of EPSPs and IPSPs analogous to that present in the OFF subfields would demand that inhibition be evoked at light-off in the ON region of this simple cell. Figure 3 shows that turning a light off in the ON region 


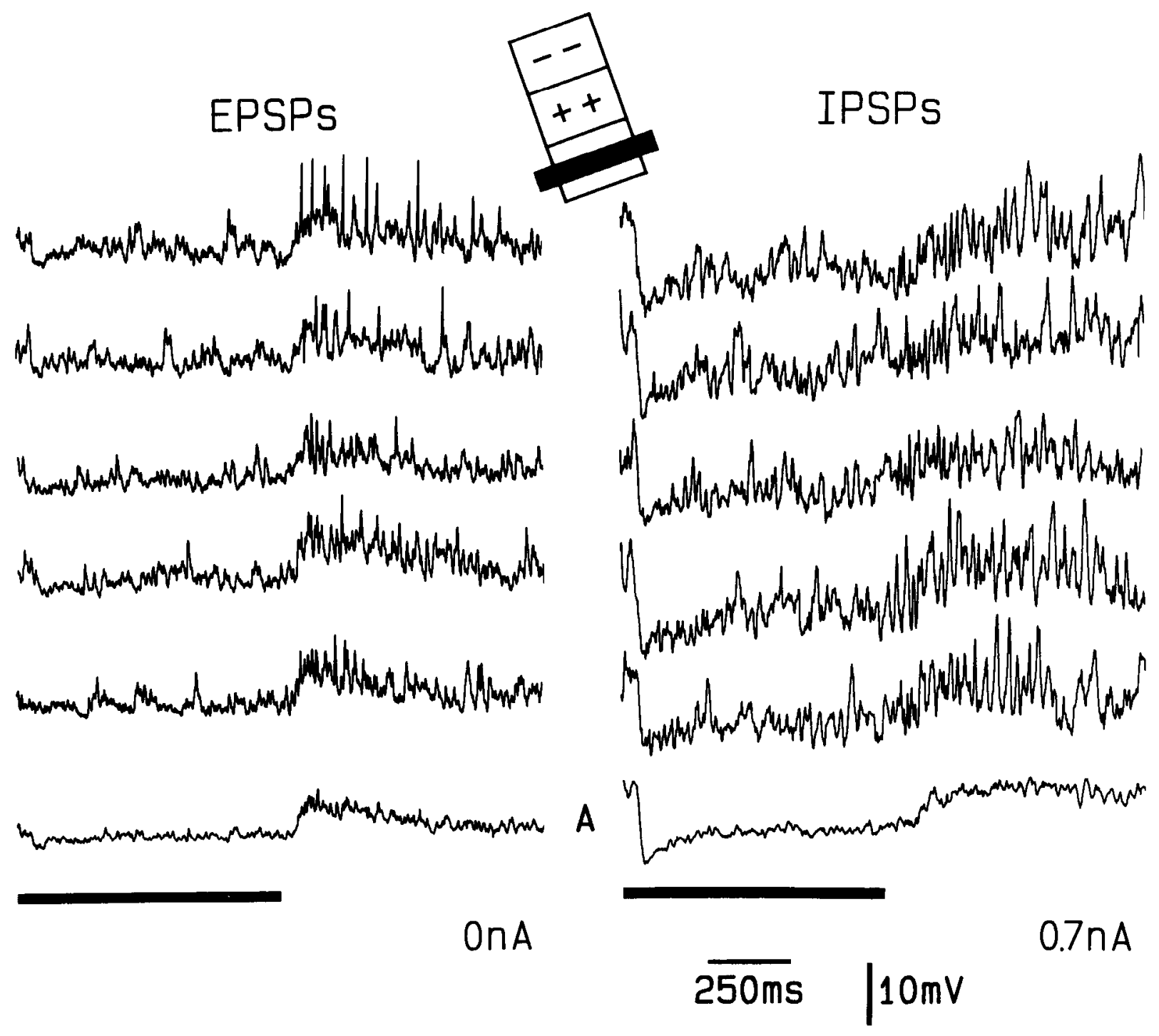

Figure 4. Responses to a bar flashed in one OFF region of the simple cell of Figures 1-3. Conventions as in Figure 3.

evoked as strong an inhibition (though a slightly more sustained one) as did turning a light on in the OFF region. As in Figure 4 , the phase of the stimulus that evoked large EPSPs, in this case turning the light off, shut off the inhibition. The latency of the inhibition evoked in both Figures 3 and 4 was $50 \mathrm{msec}$. Thus the onset of the inhibition followed the beginning of the decline of excitation by approximately $5 \mathrm{msec}$ in each case.

Though similar in shape, the EPSP and IPSP records of Figures 1,3 , and 4 differ in 3 respects:

1. In the IPSP records, the spikes have been slightly diminished in size by the current-induced depolarization, and broadened by the $\mathrm{Ce}^{+}$-induced inactivation of $\mathrm{K}^{+}$channels. These spikes occur at rest in the IPSP records, presumably because of the depolarization, and are suppressed by the stimulus-induced hyperpolarization. Thus there is an apparent decrease in the noise of the records during the inhibition, despite the increase in synaptic activity. This is not the case with records taken without $\mathrm{Ce}^{+}$injections (see Fig. 5).

2. Unlike the EPSP records, the potential in any of the IPSP records is not modulated above and below the resting level. Only hyperpolarizations from rest are present, as if there could be no withdrawal of tonic inhibition evoked by the stimulus. Activity in the presynaptic inhibitory interneurons seems to return to rest when the light is turned off in Figure 4 or on in Figure 3. The interneurons were either insensitive to these stimuli or their resting discharge level was near 0 and could not be decreased further by an antagonistic stimulus.

3. The apparent widths of the inhibitory subfields in Figure 1 are larger than those of the OFF regions in which they are centered. Thus the ON inhibition in the OFF regions overlaps partially with the central ON region (see also Heggelund, 1986). The same is likely to be true for OFF inhibition in the ON region, though this was not tested. It is also possible that the ON and OFF excitatory mechanisms overlap at the borders of the subregions, but since the EPSPs evoked by the moving stimuli reflect the sum of the $\mathrm{ON}$ and OFF inputs, any overlap would not be detectable in Figure 1.

During the study of the simple cell illustrated in Figures 14 , the bar was flashed numerous times in regions just outside the 2 OFF regions. No potentials were detected in response to such stimuli. One might expect that the surrounds of the geniculate neurons that make up the OFF regions might be de- 


\section{EPSPS}

\section{IPSPS}
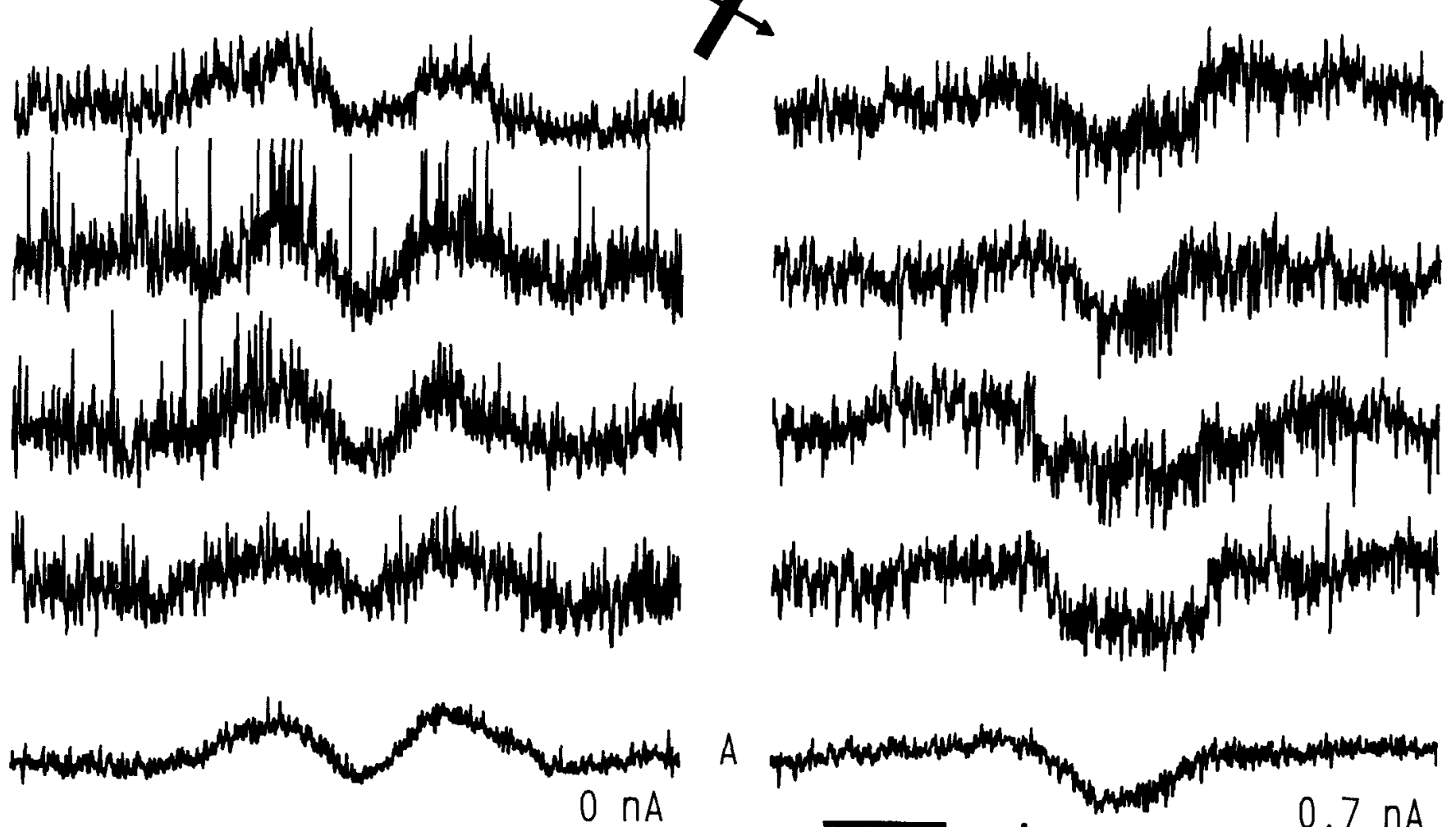

\section{A}

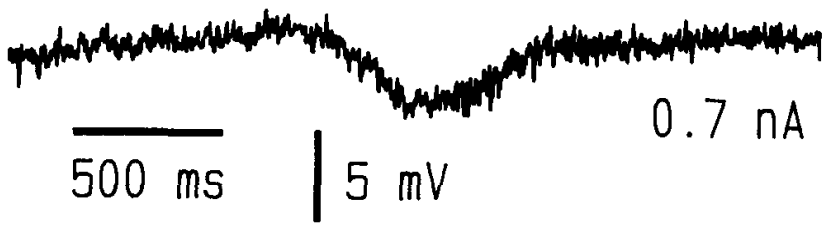

Figure 5. Responses to a moving bright bar recorded intracellularly from a second simple cell. The receptive field of this cell consisted of 2 ON regions flanking a central OFF region. The bar moved at a rate of $2 \% \mathrm{sec}$.

tectable in the EPSP records, but the effects of the surrounds were apparently too weak. Neither were any IPSPs evoked outside the 3 subfields of the receptive field. The receptive fields of the EPSPs and IPSPs were completely coextensive, as predicted by previous extracellular records (Palmer and Davis, 1981; Heggelund, 1986).

Figures 5 and 6 contain records analogous to those in Figures 1 and 2 for a second simple cell. In this cell, by coincidence,
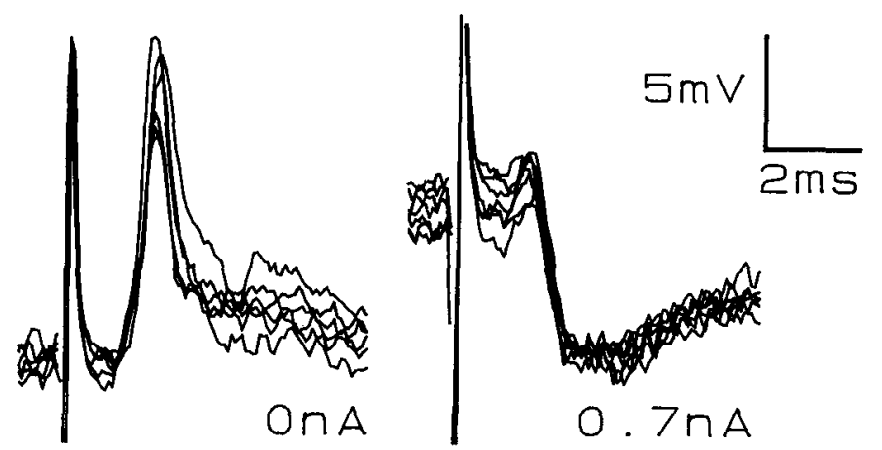

Figure 6. Synaptic potential evoked by electrical stimulation in the LGN, recorded in the same simple cell as in Figure 5. Stimulus amplitude, $1 \mathrm{~mA}$; duration, $200 \mathrm{msec}$. the levels of current required for separating EPSPs and IPSPS were also 0 and $0.7 \mathrm{nA}$ (Fig. 6). The electrode in this case was filled with $\mathrm{K}^{+}$-acetate, rather than $\mathrm{Cc}^{+}$-acetate, and only the response to a moving bar of light was recorded. In the EPSP records, the bar evoked 2 separate waves of depolarization, indicating the presence of $2 \mathrm{ON}$ regions. A hyperpolarization back to rest (and even slightly beyond) that was evoked in the region between the $2 \mathrm{ON}$ subfields indicated the presence of a central OFF region, making the receptive field of this cell the obverse of that in Figures 1-4. As for the simple cell in Figures $1-4$, sweeping the slit in the opposite direction evoked an almost identical response to that in Figure 5 (not shown).

The visually evoked IPSPs recorded with the cell depolarized consisted primarily of a single hyperpolarization evoked as the bright bar entered the OFF region. The overall shapes of the 2 averaged traces in Figure 5 are quite different from each other and suggest strongly the presence of $O N$ inhibition in the OFF region. The small upward spikes in the EPSP records of Figure 5 are similar in size and duration to those evoked by electrical stimulation in Figure 6; the downward spikes in the IPSP records of Figure 5 have a time course identical to the IPSPs evoked electrically in Figure 6, and likely represent unitary IPSPs (Ferster, 1986). Once again, the inhibitory subfield extends well beyond the OFF region on which it is centered, into the adjacent ON regions. 
$A$
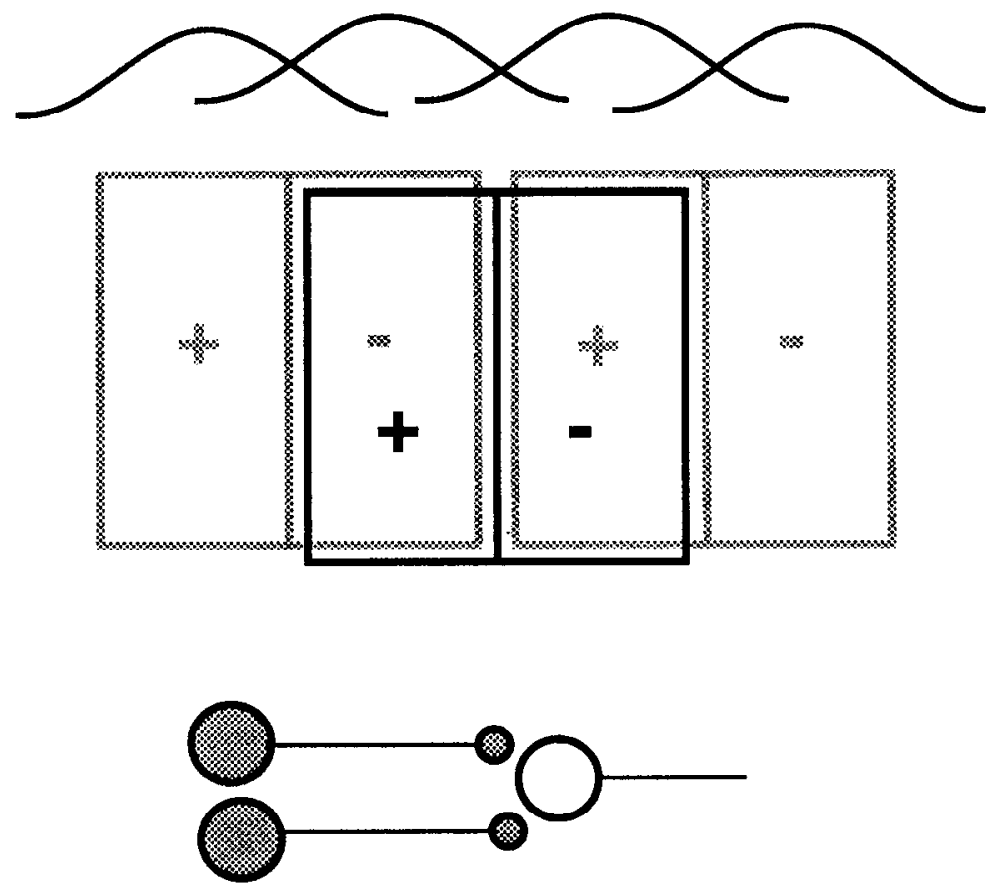

$B$
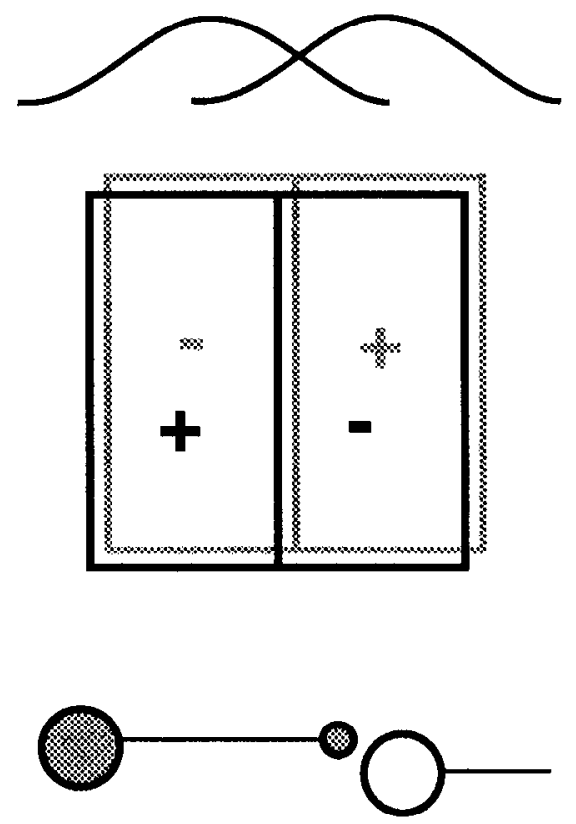

Figure 7. Alternative models of the origin of opponent inhibition in the receptive fields of simple cells. In each case it is assumed that the inhibition is provided by synaptic connections from other simple cells with subfields of identical size and shape to that of the postsynaptic cell. The receptive field of the postsynaptic cell is drawn in solid lines, and the type of each subfield, ON or OFF, is indicated by a + or - . The receptive fields of the inhibitory interneurons are shaded. The subfields of both the inhibitory interneuron and the postsynaptic cells arise from direct excitation by ON- and OFF-center geniculate neurons. $A$, The receptive fields of pre- and postsynaptic neurons overlap only partially. Such an arrangement apparently does not occur in the cortex. In the cells so far examined, no inhibition could be evoked outside the receptive field defined by the EPSPs. $B$, The receptive fields of the pre- and postsynaptic neurons are coextensive, as suggested by Palmer and Davis (1981) and by Heggelund (1986). There is also the possibility that the inhibitory interneurons have fewer subfields than the postsynaptic neuron, and that more than one interneuron is required to provide inhibition of the appropriate type to all of the subregions. For clarity, the subregions of the receptive fields are drawn with sharp borders and no overlap. Above each subfield is a broad curve that is meant to represent its true extent in the direction perpendicular to the receptive-field orientation. The intracellular records indicate that the ON inhibition is centered in each OFF region, but overlaps the adjacent ON region. Similarly, the OFF inhibition is centered in the ON region, but slightly overlaps the adjacent OFF region.

\section{Discussion}

Source of spatially opponent inhibition

Evidence has been collected from 6 simple cells, including the 2 illustrated here, that each of their subfields is constructed from 2 separate mechanisms: ON excitation and OFF inhibition on ON regions, and OFF excitation and ON inhibition in OFF regions. These IPSPs are similar in receptive-field properties to those proposed by Hubel and Wiesel (1962) to enhance the antagonism between subfields, but differ in their source, most likely arising from interneurons within the cortex rather than directly from the LGN. Several properties of the IPSPs provide information about the presynaptic inhibitory interneurons that produce them: (1) IPSPs in simple cells, as in most cortical cells, have the same preferred orientation as the excitatory drive recorded in the same cell (Fcrster, 1986). The interneurons must therefore have the same preferred orientation and reside in the same set of orientation columns as the postsynaptic cell from which the IPSPs were recorded. (2) The interneurons must possess at least one pure $\mathrm{ON}$ region, one OFF region, or both, in order to give rise to the pure ON and OFF IPSPs that were observed. (3) The number of presynaptic neurons responsible for the IPSPs is probably small, perhaps no more than 3 . As in Figure 5, unitary IPSPs are often distinguishable in IPSP records
(Ferster, 1986). These short hyperpolarizing potentials are almost identical in time course to the IPSPs evoked by electrical stimulation of the LGN. Their regular frequency and near-uniform amplitude imply that the presynaptic cells that produce them are few in number. (Unitary IPSPs are not visible in the records of Figs. 1, 3, and 4 because of the presence of $\mathrm{Ce}^{+}$; the very long time constant of the IPSPs, visible in Fig. $2 C$, makes unitary responses occurring close together in time unresolvable.) (4) That the IPSP traces in Figures 1, and 3-5 contain primarily hyperpolarizations (i.e., that little disinhibition is observed) would suggest that the presynaptic cells have little or no spontaneous activity and therefore produce no tonic inhibition that could be suppressed by an appropriate stimulus. (5) The IPSPs evoked in simple cells by electrical stimulation of the LGN are disynaptic (Ferster and Lindström, 1983). The smooth, uninflected time course of the traces taken with depolarizing current in Figures 2 and 6 indicate the absence of later di- or polysynaptic components. Assuming that the interneurons responsible for the visually evoked IPSPs are among those responsible for the electrically evoked IPSP, they must receive monosynaptic excitation from the LGN, much like the simple cells from which they were recorded.

Given these properties of the observed IPSPs, the most obvious candidates for the presynaptic inhibitory interneurons are 
other simple cells. They would be required to have subregions identical in size, shape, and orientation to those of the postsynaptic cell, but would have $\mathrm{ON}$ regions wherever the postsynaptic cell has OFF regions, and vice versa, as originally suggested by Palmer and Davis (1981).

Two possible arrangements for the receptive fields of inhibitory interneurons are shown in Figure 7 . In Figure $7 A$, the receptive fields of 2 inhibitory interneurons only partially overlap that of the postsynaptic cell. But in the intracellular records, IPSPs could never be evoked from any region outside the excitatory receptive field in any of the cells studied, including those illustrated here. Therefore, the arrangement of the interneurons' receptive field more likely resembles that illustrated in Figure $7 \mathrm{~B}$, in which the receptive field of the interneuron is entirely contained within that of the EPSPs. Palmer and Davis (1981) were able to find adjacent simple cells with overlapping receptive fields, but each pair of overlapping subfields were always of the opposite type, ON or OFF, as in Figure $7 B$. Palmer and Davis suggested that one of these neurons might be inhibitory to the other.

\section{Effects of spatially opponent inhibition on the visual responses of simple cells}

Mutual antagonism between subfields. $\Lambda$ s suggested by Hubel and Wiesel (1962), spatially opponent inhibition could contribute to antagonism between subfields (Hubel and Wiesel, 1962). Turning on a single bright bar in an ON region of the cell in Figure $7 B$ would be an excellent stimulus. An additional bar in an OFF region would antagonize the first by suppressing the spontaneous activity of excitatory geniculate OFF-center cells. The interneuron in Figure $7 B$ would not respond at all to the onset of the single bar, which would be located in its OFF region. But it might respond to the second stimulus, which would be located in its ON region. The response would be slight, because the interneuron is itself a simple cell and would experience mutual antagonism of its own. But however much the interneuron responded, it would help to suppress the response to the double stimulus and to make the postsynaptic neuron more selective for the single stimulus. That such suppression does occur seems clear from the double-slit experiments of Heggelund (1986).

Spatial frequency. The same arguments for mutual antagonism may be cast in terms of spatial frequency. A stimulus consisting of 2 slits contains larger lower-frequency components than does a single slit. The single slit activates the postsynaptic neuron strongly, but the interneuron hardly at all. Therefore, however much the double slit activated the interneuron, that activity would serve to make the postsynaptic cell more selective for higher frequencies. The inhibition could, in theory, make cortical neurons more selective for spatial frequency than for their geniculate inputs, but it is not clear when comparing the spatial frequency tuning curves of geniculate neurons (Derrington and Fuchs, 1979; Troy, 1983) with those of cortical neurons (Movshon et al., 1978; Tolhurst and Thompson, 1981) that a significant sharpening of selectivity occurs.

Orientation selectivity. The neurons that mediate spatially opponent inhibition must themselves be orientation-selective and are tuned to the same orientation as their postsynaptic targets. At orientations far from the optimal, they are largely inactive and likely to have little effect on the postsynaptic cell's responses (Ferster, 1986), but near the optimal orientation, they are in a position to alter the sharpness of tuning (Orban, 1984, see pp. 289-295). An optimally oriented bright bar that is turned on within the ON region of a simple cell will not activate inhibitory interneurons at all. But if the bar is turned only slightly, it will begin to encroach upon the flanking OFF regions and their accompanying ON inhibition. Because the ON inhibitory zone partially overlaps the $\mathrm{ON}$ region, even the slightest misalignment of the bar could begin to activate inhibitory inputs. As the stimulus is turned farther away from optimal, it will move more and more into the $\mathrm{ON}$ region of the inhibitory interneuron, but at the same time the orientation becomes less and less optimal for the inhibitory interneuron. Whether the inhibition actually affects orientation tuning will depend on the exact spatial arrangement of the subfields, on how much of the subfield must be stimulated for the interneurons to reach threshold, and on the orientation tuning of the interneurons compared to that of the excitatory input.

Disparity sensitivity. An important function of opponent inhibition in simple cells might be a contribution to disparity sensitivity. Most simple cells that possess binocular receptive fields are extremely sensitive to stimulus disparity, with their optimal disparity falling very near the horopter, or zero disparity (Ferster, 1981; Ohzawa and Freeman, 1986). As a result, they respond poorly to a stimulus of non-zero disparity, that is, to the image of a bar that falls within an $O N$ region of one eye and an OFF region of the other. The mechanism that produces disparity sensitivity in simple cells could involve both excitation and inhibition. A bright bar presented at a nonoptimal disparity, for example, might fall on the $O N$ region in the receptive field of one eye. But if it fell on an OFF region in the other eye, it could suppress the cell by virtue of the suppression of excitation from OFF-center geniculate cells. The intracellular records indicate that it would, in addition, evoke an active synaptic inhibition in the OFF region.

Time course of the response of simple cells. Finally, the reciprocal inhibition in simple cell subregions may be important for shaping the time course of a simple cell's response to flashing stimuli. The decline of excitation at light-off in Figure 4 lasts for almost $100 \mathrm{msec}$ after the excitatory stimulus is withdrawn, much like the relatively slow decline of activity in some retinal ganglion cells and geniculate relay cells (Steinberg, 1983; Cleland and Lee, 1985; Cleland et al., 1971). Visually evoked inhibition, with a latency of $50 \mathrm{msec}$, may prevent the declining EPSP from continuing to evoke action potentials, allowing the cell to represent more faithfully the time course of the stimulus. To test this possibility, careful comparisons of the time course of the responses of simple cells and of geniculate relay cells would be required. But whether the spatial or the temporal properties of the inhibition are most important, EPSPs of geniculate origin and IPSPs of cortical origin are reciprocally organized to reinforce each other's effects on the membrane potential of cortical simple cells.

\section{References}

Cleland, B. G., and B. B. Lee (1985) A comparison of visual responses of cat lateral geniculate nucleus neurones with those of the ganglion cells afferent to them. J. Physiol. (Lond.) 369: 249-268.

Cleland, B. G., M. W. Dubin, and W. R. Levick (1971) Sustained and transient neurones in the cat's retina and lateral geniculate nucleus. J. Physiol. (Lond.) 217: 473-496.

Derrington, A. M., and A. F. Fuchs (1979) Spatial and temporal properties of $\mathrm{X}$ and $\mathrm{Y}$ cells in the cat lateral geniculate nucleus. J. Physiol. (Lond.) 293: 347-364.

Ferster, D. (1981) A comparison of binocular depth mechanisms in 
areas 17 and 18 of the cat visual cortex. J. Physiol. (Lond.) 311:623655.

Ferster, D. (1986) Orientation selectivity of synaptic potentials in neurons of cat primary visual cortex. J. Neurosci. 6: 1284-1301.

Ferster, D. (1987) The origin of orientation selective EPSPs in simple cells of cat visual cortex. J. Neurosci. 7: 1780-1791.

Ferster, D., and S. Lindström (1983) An intracellular analysis of geniculocortical connectivity in area 17 of the cat. J. Physiol. (Lond.) 342: $181-215$.

Heggelund, P. (1986) Quantitative studies of enhancement and suppression zones in the receptive field of simple cells in cat striate cortex. J. Physiol. (Lond.) 373: 293-310.

Hubel, D. H. , and T. N. Wiesel (1962) Receptive fields, binocular interaction and functional architecture in the cat's visual cortex. J. Physiol. (Lond.) 160: 106-154.

Johnston, D., J. J. Hablitz, and W. A. Wilson (1980) Voltage clamp discloses slow inward current in hippocampal burst-firing neurones. Nature 286: 391-393.

Movshon, J. A., I. D. Thompson, and D. J. Tolhurst (1978) Spatial and temporal contrast sensitivity of neurones in areas 17 and 18 of the cat's visual cortex. J. Physiol. (Lond.) 283: 101-120.

Ohzawa, I., and R. D. Freeman (1986) The binocular organization of simple cells in the cat's visual cortex. J. Neurophysiol. 56: 221-242.

Orban, G. (1984) Neuronal Operations in the Visual Cortex, SpringerVerlag, Berlin, pp. 289-295.
Palmer, L. A., and T. L. Davis (1981) Receptive field structure in cat striate cortex. J. Neurophysiol. 46: 260-276.

Sillito, A. M. (1977) Inhibitory processes underlying the directional specificity of simple, complex and hypercomplex cells in the cat's visual cortex. J. Physiol. (Lond.) 27 1: 699-720.

Steinberg, R. H. (1983) High-intensity effects on slow potentials and ganglion cell activity in area centralis of cat retina. Vision Res. 9: 333-350.

Tanaka, K. (1983) Cross-correlation analysis of geniculostriate neuronal relationships in cats. J. Neurophysiol. 49: 1303-1318.

Tolhurst, D. J., and I. D. Thompson (1981) On the variety of spatial frequency selectivities shown by neurons in area 17 of the cat. Proc. R. Soc. Lond. [Biol.] 213: 183-199.

Toyama, K., K. Matsunami, T. Ohno, and S. Tokashiki (1974) An intracellular study of neuronal organization in the visual cortex. Exp. Brain Res. 21: 45-66.

Toyama, K., M. Kimura, and K. Tanaka (1981) Organization of cat visual cortex as investigated by cross-correlation technique. J. Neurophysiol. 46: 191-201.

Troy, J. B. (1983) Spatial contrast sensitivities of $X$ and $Y$ type neurones in the cat's dorsal lateral geniculate nucleus. J. Physiol. (Lond.) 344: 399-417. 